\title{
The Ladder Crystal
}

\section{Chris Berg}

University of California, Davis

One Shields Ave.

Davis, CA 95616

Abstract. In this paper, we introduce a new model of the crystal $B\left(\Lambda_{0}\right)$ of $\widehat{\mathfrak{s l}_{\ell}}$. We briefly describe some of the properties of this crystal and compare it to the combinatorial model of Misra and Miwa.

Résumé. Dans cet article on propose un nouveau modèle du cristal $B\left(\Lambda_{0}\right)$ de $\widehat{\mathfrak{s l}_{\ell}}$. On décrit brièvement les propriétés du cristal et on le compare avec le modèle combinatoire de Misra et Miwa.

Keywords: combinatorics of Young diagrams, crystals, representation theory of Hecke algebras

\section{Introduction}

One description of the crystal $B\left(\Lambda_{0}\right)$ of $\widehat{\mathfrak{s l}_{\ell}}$ has as nodes $\ell$-regular partitions. In this paper we give another combinatorial description of $B\left(\Lambda_{0}\right)$, called the ladder crystal, which we denote $B\left(\Lambda_{0}\right)^{L}$. Our crystal satisfies the following properties:

- The nodes of $B\left(\Lambda_{0}\right)^{L}$ are partitions, and there is an $i$-arrow from $\lambda$ to $\mu$ only when the difference $\mu \backslash \lambda$ is a box of residue $i$.

- There exists elementary combinatorial arguments which generalize crystal theoretic results of $B\left(\Lambda_{0}\right)$ to $B\left(\Lambda_{0}\right)^{L}$.

- $B\left(\Lambda_{0}\right) \cong B\left(\Lambda_{0}\right)^{L}$ and the isomorphism is a well studied (but never before in this context) map on partitions.

- The nodes of $B\left(\Lambda_{0}\right)^{L}$ have a simple combinatorial description.

The new description of the crystal $B\left(\Lambda_{0}\right)$ is in many ways more important than the theorems which were proven by the existence of it. Besides the fact that it is a useful tool in proving theorems about $B\left(\Lambda_{0}\right)$, our new description also highlights a set of partitions (in bijection to $\ell$-regular partitions), which can be interpreted in terms of the representation theory of the finite Hecke algebra $H_{n}(q)$.

Remark 1.0.1 All proofs are absent from this text in the interest of space, as several of them require tedious calculations.

1365-8050 @ 2009 Discrete Mathematics and Theoretical Computer Science (DMTCS), Nancy, France 


\subsection{Combinatorial definitions on partitions}

Let $\lambda$ be a partition of $n$ (written $\lambda \vdash n$ ) and $\ell \geq 3$ be an integer. We will use the convention $(x, y)$ to denote the box which sits in the $x^{\text {th }}$ row and the $y^{\text {th }}$ column of the Young diagram of $\lambda$. We denote the transpose of $\lambda$ by $\lambda^{\prime}$. Throughout this paper, all of our partitions are drawn in English notation. An $\ell$-regular partition is one in which no part occurs $\ell$ or more times.

The hook length of the $(a, c)$ box of $\lambda$ is defined to be the number of boxes to the right and below the box $(a, c)$, including the box $(a, c)$ itself. It will be denoted $h_{(a, c)}^{\lambda}$. The arm of the $(a, c)$ box of $\lambda$ is defined to be the number of boxes to the right of the box $(a, c)$, not including the box $(a, c)$. It will be denoted $\operatorname{arm}(a, c)$. Similarly, the leg is below $(a, c)$, not including $(a, c)$ and will be denoted $\operatorname{leg}(a, c)$.

Remark 1.1.1 From the definitions, it is clear that $h_{(a, c)}^{\lambda}=\operatorname{arm}(a, c)+\operatorname{leg}(a, c)+1$.

\section{Hecke Algebras}

\subsection{Representation theory of $H_{n}(q)$}

Definition 2.1.1 For a fixed field $\mathbb{F}$ of characteristic zero and $0 \neq q \in \mathbb{F}$, the finite Hecke Algebra $H_{n}(q)$ is defined to be the $\mathbb{F}$-algebra generated by $T_{1}, \ldots, T_{n-1}$ with relations

$$
\begin{array}{ll}
T_{i} T_{j}=T_{j} T_{i} & \text { for }|i-j|>1 \\
T_{i} T_{i+1} T_{i}=T_{i+1} T_{i} T_{i+1} & \text { for } 1 \leq i<n-1 \\
T_{i}^{2}=(q-1) T_{i}+q & \text { for } 1 \leq i \leq n-1 .
\end{array}
$$

In this paper we will always assume that $q \neq 1$ and that $q \in \mathbb{F}$ is a primitive $\ell^{t h}$ root of unity in a field $\mathbb{F}$ of characteristic zero (so necessarily $\ell \geq 2$ ).

Remark 2.1.2 When $q$ is specialized to 1 the Hecke algebra becomes the group algebra of the symmetric group.

Similar to the symmetric group, a construction of the Specht module $S^{\lambda}=S^{\lambda}[q]$ exists for $H_{n}(q)$ (see (2)). The Specht modules need not remain irreducible when $q$ is a primitive $\ell^{t h}$ root of unity. Conditions for the irreducibility of these modules was conjectured by James and Mathas, and recently proven in work of Fayers (3) and Lyle (11).

All of the irreducible representations of $H_{n}(q)$ have been constructed when $q$ is a primitive $\ell$ th root of unity. These modules are indexed by $\ell$-regular partitions $\lambda$, and are called $D^{\lambda} . D^{\lambda}$ is the unique simple quotient of $S^{\lambda}$ (see (2) for more details). In particular $D^{\lambda}=S^{\lambda}$ if and only if $S^{\lambda}$ is irreducible and $\lambda$ is $\ell$-regular. For $\lambda$ not necessarily $\ell$-regular, $S^{\lambda}$ is irreducible if and only if there exists an $\ell$-regular partition $\mu$ so that $S^{\lambda} \cong D^{\mu}$.

\section{Misra-Miwa Description of $B\left(\Lambda_{0}\right)$}

\subsection{Introduction}

In this section, we recall a description of the crystal graph $B\left(\Lambda_{0}\right)$ currently used in the literature, first described by Misra and Miwa (13) . 


\subsection{Classical description of the crystal $B\left(\Lambda_{0}\right)$}

We will assume some familiarity with the theory of crystals (see (8) for details). We will look at the crystal $B\left(\Lambda_{0}\right)$ of the irreducible highest weight module $V\left(\Lambda_{0}\right)$ of the affine Lie algebra $\widehat{\mathfrak{s l}}_{\ell}$ (also called the basic representation of $\left.\widehat{\mathfrak{s l}_{\ell}}\right)$. In the model of $B\left(\Lambda_{0}\right)$ given by Misra and Miwa, the nodes are $\ell$-regular partitions. The set of nodes will be denoted $B:=\{\lambda \in \mathcal{P}: \lambda$ is $\ell$-regular $\}$. We will describe the arrows of $B\left(\Lambda_{0}\right)$ below.

We view the Young diagram for $\lambda$ as a set of boxes, with their corresponding residues $b-a \bmod \ell$ written into the box $(a, b)$. A box in $\lambda$ is said to be a removable $i$-box if it has residue $i$ and after removing that box the remaining diagram is still a partition. A space not in $\lambda$ is an addable $i$-box if it has residue $i$ and adding that box to $\lambda$ yields a partition.

Example 3.2.1 Let $\lambda=(8,5,4,1)$ and $\ell=3$. Then the residues are filled into the corresponding Young diagram as follows:



Here $\lambda$ has two removable 0 -boxes (boxes $(2,5)$ and $(4,1)$ ), two removable 1 -boxes (boxes $(1,8)$ and $(3,4))$, no removable 2-boxes, no addable 0-boxes, two addable 1-boxes (in positions $(2,6)$ and $(4,2))$, and three addable 2-boxes (in positions (1,9), (3,5) and (5,1)).

For a fixed $i,(0 \leq i<\ell)$, we place - in each removable $i$-box and + in each addable $i$-box. The $i$-signature of $\lambda$ is the word of + and - 's in the diagram for $\lambda$, written from bottom left to top right. The reduced $i$-signature is the word obtained after repeatedly removing from the $i$-signature all adjacent pairs -+ . The resulting word will now be of the form $+\cdots+++---\cdots-$. The boxes corresponding to -'s in the reduced $i$-signature are called normal $i$-boxes, and the boxes corresponding to + 's are called conormal $i$-boxes. $\varepsilon_{i}(\lambda)$ is defined to be the number of normal $i$-boxes of $\lambda$, and $\varphi_{i}(\lambda)$ is defined to be the number of conormal $i$-boxes. If a leftmost - exists, the box corresponding to such a - is called the good $i$-box of $\lambda$. If a rightmost + exists, the box corresponding to such a + is called the cogood $i$-box. All of these definitions can be found in Kleshchev's book (9).

Example 3.2.2 Let $\lambda=(8,5,4,1)$ and $\ell=3$ be as above. Fix $i=1$. The diagram for $\lambda$ with removable and addable 1-boxes marked is:



The 1-signature of $\lambda$ is +-+- , so the reduced 1-signature is $+\quad-$ and the diagram has a good 1-box in the first row, and a cogood 1-box in the fourth row. Here $\varepsilon_{1}(\lambda)=1$ and $\varphi_{1}(\lambda)=1$. 
We recall the action of the crystal operators on $B$. The crystal operator $\widetilde{e}_{i}: B \stackrel{i}{\rightarrow} B \cup\{0\}$ assigns to a partition $\lambda$ the partition $\widetilde{e}_{i}(\lambda)=\lambda \backslash x$, where $x$ is the good $i$-box of $\lambda$. If no such box exists, then $\widetilde{e}_{i}(\lambda)=0$. It is clear then that $\varepsilon_{i}(\lambda)=\max \left\{k: \widetilde{e}_{i}^{k} \lambda \neq 0\right\}$.

Similarly, $\widetilde{f}_{i}: B \stackrel{i}{\rightarrow} B \cup\{0\}$ is the operator which assigns to a partition $\lambda$ the partition $\widetilde{f}_{i}(\lambda)=\lambda \cup x$, where $x$ is the cogood $i$-box of $\lambda$. If no such box exists, then $\widetilde{f}_{i}(\lambda)=0$. It is clear then that $\varphi_{i}(\lambda)=$ $\max \left\{k: \widetilde{f}_{i}^{k} \lambda \neq 0\right\}$.

For $i \in \mathbb{Z} / \ell \mathbb{Z}$, we write $\lambda \stackrel{i}{\rightarrow} \mu$ to stand for $\widetilde{f}_{i} \lambda=\mu$. We say that there is an $i$-arrow from $\lambda$ to $\mu$. Note that $\lambda \stackrel{i}{\rightarrow} \mu$ if and only if $\widetilde{e}_{i} \mu=\lambda$. A maximal chain of consecutive $i$-arrows will be called an $i$-string. We note that the empty partition $\emptyset$ is the unique highest weight node of the crystal ( i.e. $\widetilde{e}_{i} \emptyset=0$ for every $i \in \mathbb{Z} / \ell \mathbb{Z})$ and that $B\left(\Lambda_{0}\right)$ is connected. For a picture of a part of this crystal graph, see (10) for the cases $\ell=2$ and 3 .

Example 3.2.3 Continuing with the above example, we see that $\widetilde{e}_{1}(8,5,4,1)=(7,5,4,1)$ and $\widetilde{f}_{1}(8,5,4,1)=(8,5,4,2)$. Also, $\widetilde{e}_{1}^{2}(8,5,4,1)=0$ and $\widetilde{f}_{1}^{2}(8,5,4,1)=0$. The sequence $(7,5,4,1) \stackrel{1}{\rightarrow}$ $(8,5,4,1) \stackrel{1}{\rightarrow}(8,5,4,2)$ is a 1-string of length 3 .

\section{The Ladder Crystal: $B\left(\Lambda_{0}\right)^{L}$}

\subsection{Ladders}

We first recall what a ladder is in regards to a partition. Let $\lambda$ be a partition and let $\ell>2$ be a fixed integer. For any box $(a, b)$ in the Young diagram of $\lambda$, the ladder of $(a, b)$ is the set of all positions $(c, d)$ which satisfy $\frac{c-a}{d-b}=\ell-1$ and $c, d>0$.

Remark 4.1.1 The definition implies that two boxes in the same ladder will share the same residue. An $i$-ladder will be a ladder all of whose boxes have residue $i$.

Example 4.1.2 Let $\lambda=(3,3,1), \ell=3$. Then there is a 1-ladder which contains the boxes $(1,2)$ and $(3,1)$, and a different 1-ladder which has the box $(2,3)$ in $\lambda$ and the boxes $(4,2)$ and $(6,1)$ not in $\lambda$. In the picture below, lines are drawn through the different 1-ladders.

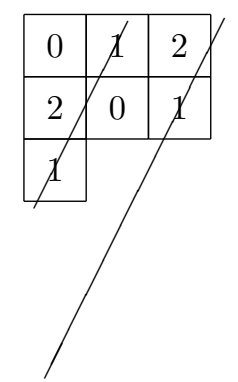

\subsection{The ladder crystal}

We will construct a new crystal $B\left(\Lambda_{0}\right)^{L}$ recursively as follows. First, the empty partition $\emptyset$ is the unique highest weight node of our crystal. From $\emptyset$, we will build the crystal by applying the operators $\widehat{f}_{i}$ for $0 \leq i<\ell$. We define $\widehat{f}_{i}$ to act on partitions, taking a partition of $n$ to a partition of $n+1$ (or to 0 ) in the 
following manner. Given $\lambda \vdash n$, first draw all of the $i$-ladders of $\lambda$ onto its Young diagram. Label any addable $i$-box with a + , and any removable $i$-box with a - . Now, write down the word of + 's and - 's by reading from leftmost $i$-ladder to rightmost $i$-ladder and reading from top to bottom on each ladder. This is called the ladder $i$-signature of $\lambda$. From here, cancel any adjacent -+ pairs in the word, until you obtain a word of the form $+\cdots+-\cdots-$. This is called the reduced ladder $i$-signature of $\lambda$. All boxes associated to a - in the reduced ladder $i$-signature are called ladder normal $i$-boxes and all boxes associated to $\mathrm{a}+$ in the reduced ladder $i$-signature are called ladder conormal $i$-boxes. The box associated to the leftmost - is called the ladder good $i$-box and the box associated to the rightmost + is called the ladder cogood $i$-box. Then we define $\widehat{f}_{i} \lambda$ to be the partition $\lambda$ union the ladder $\operatorname{cogood} i$-box. If no such box exists, then $\widehat{f}_{i} \lambda=0$. Similarly, $\widehat{e}_{i} \lambda$ is the partition $\lambda$ with the ladder good $i$-box removed. If no such box exists, then $\widehat{e}_{i} \lambda=0$. We then define $\widehat{\varphi}_{i}(\lambda)$ to be the number of ladder conormal $i$-boxes of $\lambda$ and $\widehat{\varepsilon}_{i}(\lambda)$ to be the number of ladder normal $i$-boxes. It is then obvious that $\widehat{\varphi}_{i}(\lambda)=\max \left\{k: \widehat{f}_{i}^{k} \lambda \neq 0\right\}$ and that $\widehat{\varepsilon_{i}}(\lambda)=\max \left\{k: \widehat{e}_{i}^{k} \lambda \neq 0\right\}$.

Example 4.2.1 Let $\lambda=(5,3,1,1,1,1,1)$ and $\ell=3$. Then there are four addable 2-boxes for $\lambda$. In the leftmost 2-ladder (containing box (2,1)) there are no addable (or removable) 2-boxes. In the next 2-ladder (containing box (1,3)) there is an addable 2-box in box (3,2). In the next 2-ladder (containing box $(2,4))$, there are two addable 2-boxes, in boxes $(2,4)$ and $(8,1)$. In the last drawn 2-ladder (containing box $(1,6))$ there is one addable 2-box, in box $(1,6)$. There are no removable 2-boxes in $\lambda$. Therefore the ladder 2-signature (and hence reduced ladder 2-signature) of $\lambda$ is $+_{(3,2)}+{ }_{(2,4)}+{ }_{(8,1)}+{ }_{(1,6)}$ (Here, we have included subscripts on the + signs so that the reader can see the correct order of the + 's). Hence $\widehat{f_{2}} \lambda=(6,3,1,1,1,1,1),\left(\widehat{f}_{2}\right)^{2} \lambda=(6,3,1,1,1,1,1,1),\left(\widehat{f}_{2}\right)^{3} \lambda=(6,4,1,1,1,1,1,1)$ and $\left(\widehat{f}_{2}\right)^{4} \lambda=$ $(6,4,2,1,1,1,1,1) \cdot\left(\widehat{f}_{2}\right)^{5} \lambda=0$.



Remark 4.2.2 The weight function of this crystal is exactly the same as the weight function for $B\left(\Lambda_{0}\right)$. Explicitly, the weight of $\lambda$ is $\Lambda_{0}-\sum c_{i} \alpha_{i}$ where $c_{i}$ is the number of boxes of $\lambda$ with residue $i$ (or equivalently, $\left.\sum_{i}\left(\varphi_{i}-\varepsilon_{i}\right) \Lambda_{i}\right)$. Throughout this paper we will suppress the weight function as it is irrelevant to the combinatorics involved. 


\section{Regularization}

\subsection{The operation of regularization}

In this section we describe a map from the set of partitions to the set of $\ell$-regular partitions. The map is called regularization and was first defined by James (see (6)). For a given $\lambda$, and for a ladder $\mathcal{L}$ in $\lambda$, one can count the number of boxes $\eta_{\mathcal{L}}$ on $\mathcal{L}$. Create a new partition where on ladder $\mathcal{L}$ the top $\eta_{\mathcal{L}}$ positions in $\mathcal{L}$ have boxes and all other positions on $\mathcal{L}$ are vacant. The result is called the regularization of $\lambda$, and is denoted $\mathcal{R}_{\ell} \lambda$. It can also be thought of as pushing all boxes in each ladder of $\lambda$ as far up their respective ladders as is possible. Although $\mathcal{R}_{\ell}$ depends on $\ell$, we will usually just write $\mathcal{R}$. The following theorem contains facts about regularization originally due to James (6) (see also (7)).

Theorem 5.1.1 Let $\lambda$ be a partition. Then

- $\mathcal{R} \lambda$ is $\ell$-regular

- $\mathcal{R} \lambda=\lambda$ if and only if $\lambda$ is $\ell$-regular.

- If $\lambda$ is $\ell$-regular and $D^{\lambda} \cong S^{\nu}$ for some partition $\nu$, then $\mathcal{R} \nu=\lambda$.

Regularization provides us with an equivalence relation on the set of partitions. Specifically, we say $\lambda \sim \mu$ if $\mathcal{R} \lambda=\mathcal{R} \mu$. The equivalence classes are called regularization classes, and the class of a partition $\lambda$ is denoted $\mathcal{R C}(\lambda):=\{\mu \in \mathcal{P}: \mathcal{R} \mu=\mathcal{R} \lambda\}$.

Example 5.1.2 Let $\lambda=(2,2,2,1,1,1)$ and let $\ell=3$. Then $\mathcal{R} \lambda=(3,3,2,1)$. Also,

$$
\begin{aligned}
\mathcal{R C}(\lambda)= & \{(2,2,2,1,1,1),(2,2,2,2,1),(3,2,1,1,1,1), \\
& (3,2,2,2),(3,3,1,1,1),(3,3,2,1)\}
\end{aligned}
$$

\begin{tabular}{|c|c|c|c|c|c|}
\hline 0 & 1 & \multirow[t]{6}{*}{$\mathcal{R}$} & 0 & 1 & 2 \\
\hline 2 & 0 & & 2 & 0 & 1 \\
\hline 1 & \multirow[t]{4}{*}{2} & & 1 & 2 & \\
\hline 0 & & & 0 & & \\
\hline 2 & & & & & \\
\hline 1 & & & & & \\
\hline
\end{tabular}

\section{Deregularization}

The goal of this section is to provide an algorithm for finding the smallest partition in dominance order in a given regularization class. It is nontrivial to show that a smallest partition exists. We use this result to show that our new description of the crystal $B\left(\Lambda_{0}\right)^{L}$ has nodes which are smallest in dominance order in their regularization class. All of the work of this section is joint with Brant Jones of UC Davis, who gave the first definition of a locked box. 


\subsection{Locked Boxes}

We recall a partial ordering on the set of partitions of $n$. For two partitions $\lambda$ and $\mu$ of $n$, we say that $\lambda \leq \mu$ if $\sum_{j=1}^{i} \lambda_{j} \leq \sum_{j=1}^{i} \mu_{j}$ for all $i$. This order is usually called the dominance order.

Finding all of the partitions which belong to a regularization class is not easy. The definition of locked boxes below formalizes the concept that some boxes in a partition cannot be moved down their ladders if one requires that the new diagram remain a partition.

Definition 6.1.1 For a partition $\lambda$, we label boxes of $\lambda$ as locked by the following procedure:

I. If a box $x$ has a locked box directly above it (or is on the first row) and every unoccupied space in the same ladder as $x$, lying below $x$, has an unoccupied space directly above it then $x$ is locked. Boxes locked for this reason are called type I locked boxes.

II. If a box $y$ is locked, then every box to the left of $y$ in the same row is also locked. Boxes locked for this reason are called type II locked boxes.

Boxes which are not locked are called unlocked.

Remark 6.1.2 Locked boxes can be both type I and type II.

Example 6.1.3 Let $\ell=3$ and let $\lambda=(6,5,4,3,1,1)$. Then labeling the locked boxes for $\lambda$ with an $L$ and the unlocked boxes with a $U$ yields the picture below.

\begin{tabular}{|c|c|c|c|c|c|}
\hline$L$ & $L$ & $L$ & $U$ & $U$ & $U$ \\
\hline$L$ & $L$ & $L$ & $U$ & $U$ & \\
\hline$L$ & $L$ & $U$ & $U$ & & \\
\hline$L$ & $L$ & $U$ & & & \\
\hline$L$ & & & & & \\
\hline$L$ & & & & & \\
\hline
\end{tabular}

\subsection{Algorithm for finding the smallest partition in a regularization class}

The algorithm from here is simple. For any partition $\lambda$, to find the smallest partition (with respect to dominance order) in a regularization class we first label each box of $\lambda$ as either locked or unlocked as above. Then we move all of the unlocked boxes in each ladder to the lowest unoccupied positions on their ladder. The resulting partition will be denoted $\mathcal{S} \lambda$. It is unclear that this algorithm will yield the smallest partition in $\mathcal{R C}(\lambda)$, or even that it is a partition. The following theorem resolves these issues.

Theorem 6.2.1 $\mathcal{S} \lambda$ is the unique smallest partition in its regularization class with respect to dominance order. It can be classified as being the unique partition (in its regularization class) which has all its boxes locked.

Example 6.2.2 Continuing from the example above $(\lambda=(6,5,4,3,1,1)$ and $\ell=3)$, we move all of the unlocked boxes down to obtain the smallest partition in $\mathcal{R C}(\lambda)$, which is:

$$
\mathcal{S} \lambda=(3,3,2,2,2,2,2,1,1,1,1) .
$$

The boxes labeled $L$ are the ones which were locked in $(6,5,4,3,1,1)$ (and did not move). 


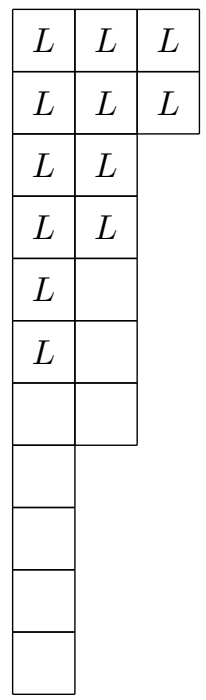

\subsection{The nodes of $B\left(\Lambda_{0}\right)^{L}$ are smallest in dominance order}

The nodes of $B\left(\Lambda_{0}\right)^{L}$ have been defined recursively by applying the operators $\widehat{f}_{i}$. We now give a simple description which determines when a partition is a node of $B\left(\Lambda_{0}\right)^{L}$.

Proposition 6.3.1 Let $\lambda$ be a partition of $n$. Let $\mathcal{R C}(\lambda)$ be its regularization class. Then $\lambda$ is a node of $B\left(\Lambda_{0}\right)^{L}$ if and only if $\lambda$ is the smallest partition in $\mathcal{R C}(\lambda)$ with respect to dominance order.

One can view $B\left(\Lambda_{0}\right)$ as having nodes $\{\mathcal{R C}(\lambda): \lambda \vdash n, n \geq 0\}$. The usual model of $B\left(\Lambda_{0}\right)$ takes the representative $\mathcal{R} \lambda \in \mathcal{R C}(\lambda)$, which happens to be the largest in dominance order. Here, we will take a different representative of $\mathcal{R C}(\lambda)$, the partitions $\mathcal{S} \lambda$, which are smallest in dominance order.

\section{Crystal Isomorphism}

\subsection{The isomorphism $B\left(\Lambda_{0}\right) \cong B\left(\Lambda_{0}\right)^{L}$}

Using the theory of locked boxes described above, we were able to prove the following theorem.

Theorem 7.1.1 Regularization commutes with the crystal operators. In other words if $\lambda \in B\left(\Lambda_{0}\right)^{L}$ then:

1. $\left(\mathcal{R} \circ \widehat{f}_{i}\right)(\lambda)=\left(\tilde{f}_{i} \circ \mathcal{R}\right)(\lambda)$,

2. $\left(\mathcal{R} \circ \widehat{e}_{i}\right)(\lambda)=\left(\tilde{e}_{i} \circ \mathcal{R}\right)(\lambda)$.

A corollary to this theorem is that the crystals are isomorphic, the isomorphism being regularization in one direction. The inverse to regularization is the map $\mathcal{S}$ described above.

Corollary 7.1.2 The crystal $B\left(\Lambda_{0}\right)$ is isomorphic to $B\left(\Lambda_{0}\right)^{L}$.

Example 7.1.3 Let $\lambda=(2,1,1,1)$ and $\ell=3$. Then $\mathcal{R} \lambda=(2,2,1)$. Also $\widehat{f}_{2} \lambda=(2,1,1,1,1)$ and $\tilde{f}_{2}(2,2,1)=(3,2,1)$. But $\mathcal{R}(2,1,1,1,1)=(3,2,1)$. 


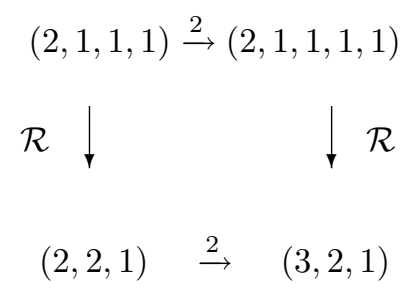

\section{The Mullineux Map}

The operation on the category of $H_{n}(q)$ modules of tensoring with the sign module (the 1-dimensional module of $H_{n}(q)$ where each $T_{i}$ acts as -1$)$ is a functor which takes irreducible modules to irreducible modules. For instance, when $q$ is not a root of unity, then $S^{\lambda} \otimes \operatorname{sign}=S^{\lambda^{\prime}}$, where $\lambda^{\prime}$ is the transpose of $\lambda$. When $\lambda$ is an $\ell$-regular partition, and $D^{\lambda}$ denotes the irreducible module corresponding to $\lambda$ then $D^{\lambda} \otimes$ sign is some irreducible module $D^{m(\lambda)}$. This describes a map $m$ between $\ell$-regular partitions called the Mullineux map. Recent results of Fayers (4) settle a conjecture of Lyle (12) which effectively computes the Mullineux map in certain cases by means of regularization and transposition. This section will highlight the interpretation of Fayers result in terms of the ladder crystal. It should be noted that Ford and Kleshchev gave a recursive construction for computing the Mullineux map in all cases (5).

\subsection{Connections with Fayers results}

Since the Mullineux map is the modular analog of transposition, a natural attempt to compute $m(\lambda)$ for an $\ell$-regular partition $\lambda$ would be to transpose $\lambda$ and then regularize. If a partition is not $\ell$-regular, we could similarly guess that $m(\mathcal{R} \lambda)$ was just transposing $\lambda$ and then regularize the result. This is not always the case. However, a conjecture of Lyle (12), which was proven recently by Fayers (4) gives a precise classification for when this holds. The definition below was taken from Fayers (4).

Definition 8.1.1 An L-partition is a partition which has no box $(i, j)$ in the diagram of $\lambda$ such that $\ell \mid h_{i, j}^{\lambda}$ and either $\operatorname{arm}(i, j)<(\ell-1) \operatorname{leg}(i, j) \operatorname{or} \operatorname{leg}(i, j)<(\ell-1) \operatorname{arm}(i, j)$.

Theorem 8.1.2 (Fayers (4)) A partition is an L-partition if and only if $m(\mathcal{R} \lambda)=\mathcal{R} \lambda^{\prime}$.

It was pointed out to the author by Fayers that a classification of the nodes of $B\left(\Lambda_{0}\right)^{L}$ can be described in terms of hook lengths and arm lengths. We now include this classification.

Theorem 8.1.3 A partition $\lambda$ belongs to the crystal $B\left(\Lambda_{0}\right)^{L}$ if and only if there does not exist a box $(i, j)$ in the Young diagram of $\lambda$ such that $h_{(i, j)}^{\lambda}=\ell * \operatorname{arm}(i, j)$.

This classification of the partitions in $B\left(\Lambda_{0}\right)^{L}$ implies the following theorem.

Theorem 8.1.4 All L-partitions are nodes of the crystal $B\left(\Lambda_{0}\right)^{L}$.

It is easy to show that any partition $\lambda$ for which the Specht module $S^{\lambda}$ is irreducible is an L-partition. This implies the following corollary.

Corollary 8.1.5 All partitions $\lambda$ for which $S^{\lambda}$ is irreducible (when $q$ is an $\ell^{\text {th }}$ root of unity) are nodes of the crystal $B\left(\Lambda_{0}\right)^{L}$. 


\section{Conclusion}

We have built a model of the crystal $B\left(\Lambda_{0}\right)$ which has different partitions representing each regularization class. It has the surprising property that every partition $\lambda$ for which the Specht module $S^{\lambda}$ is irreducible appears. Other results relating to the representation theory of $H_{n}(q)$ and the crystal $B\left(\Lambda_{0}\right)$ can be obtained using the isomorphism between $B\left(\Lambda_{0}\right)$ and $B\left(\Lambda_{0}\right)^{L}$. In particular, generalizations of theorems from (1) can be proven with the use of $B\left(\Lambda_{0}\right)^{L}$. We have left these out to save space, but can be found in the authors upcoming thesis.

\section{References}

[1] C. Berg and M. Vazirani, $(\ell, 0)$-Carter partitions, a generating function, and their crystal theoretic interpretation, ArXiv Mathematics e-prints, math.CO/0712.2075.

[2] R. Dipper and G. James, Representations of Hecke algebras of general linear groups, Proc. LMS (3), 52 (1986), 20-52

[3] M. Fayers, Irreducible Specht modules for Hecke Algebras of Type A, Adv. Math. 193 (2005) 438452

[4] M. Fayers, Regularisation and the Mullineux map, ArXiv Mathematics e-prints, math.CO/0808.1679

[5] B. Ford and A. Kleshchev, A proof of the Mullineux conjecture, Math. Z. 226 (1997), 267-308.

[6] G.D. James, The decomposition matrices of $G L_{n}(q)$ for $n \leq 10$, Proc. Lond. Math. Soc. (3), 60 (1990), 225-264.

[7] G.D. James and A. Mathas, A q-analogue of the Jantzen-Schaper theorem, Proc. Lond. Math. Soc., 74 (1997), 241-274.

[8] M. Kashiwara, On crystal bases, in Representations of groups (Banff 1994), CMS Conf. Proc. 16 (1995), 155-197.

[9] A. Kleshchev, Linear and Projective Representations of Symmetric Groups, Cambridge Tracts in Mathematics 163.

[10] A. Lascoux, B. Leclerc, and J.-Y. Thibon, Hecke algebras at roots of unity and crystal bases of quantum affine algebras, Comm. Math. Phys. 181 (1996), 205-263.

[11] S. Lyle, Some q-analogues of the Carter-Payne Theorem, J. Reine Angew. Math., to appear

[12] S. Lyle, Some topics in the representation theory of the symmetric and general linear groups, Ph.D. thesis, University of London, 2003.

[13] K.C. Misra and T. Miwa, Crystal base for the basic representation of $U_{q}\left(\mathfrak{s l}_{n}\right)$, Commun. Math. Phys. 134 (1990), 79-88. 